\title{
Rapid variability of accretion in AM Herculis
}

\author{
D. de Martino ${ }^{1}$, G. Matt ${ }^{2}$, B. T. Gänsicke ${ }^{3}$, R. Silvotti ${ }^{1}$, J. M. Bonnet-Bidaud ${ }^{4}$, and M. Mouchet ${ }^{5,6}$ \\ 1 Osservatorio Astronomico di Capodimonte, via Moiariello 16, 80131 Napoli, Italy \\ e-mail: demartino@na.astro.it; silvotti@na.astro.it \\ 2 Dipartimento di Fisica, Universita' degli Studi Roma Tre, via della Vasca Navale, Roma, Italy \\ e-mail: matt@fis.uniroma3.it \\ 3 Astronomy Group, University of Southampton, Hampshire, UK \\ e-mail: btg@astro.soton.ac.uk \\ ${ }^{4}$ Service d'Astrophysique Saclay, Gif-Sur-Yvette, France \\ e-mail: bobi@discovery.saclay.cea.fr \\ ${ }^{5}$ LUTH FRE2462 du CNRS, Observatoire de Paris, Section de Meudon, 92195 Meudon Cedex, France \\ ${ }^{6}$ Université Denis Diderot, Place Jussieu, 75005 Paris, France \\ e-mail: martine.mouchet@obspm.fr
}

Received 25 July 2002 / Accepted 5 September 2002

\begin{abstract}
We present the last pointed observation of AM Her carried out during the life of the BeppoSAX satellite. It was bright at the beginning of the observation, but dropped to the lowest X-ray level ever observed so far. The X-ray emission during the bright period is consistent with accretion occurring onto the main pole of the magnetized white dwarf. The rapid change from the active state to the low deep state indicates a drop by a factor of 17 in the accretion rate and hence that accretion switchedoff. The short timescale (less than one hour) of this variation still remains a puzzle. Optical photometry acquired simultaneousy during the low state shows that the white dwarf remains heated, although a weak emission from the accretion stream could be still present. Cyclotron radiation, usually dominating the $V$ and $R$ bands, is negligible thus corroborating the possibility that AM Her was in an off-accretion state. The X-ray emission during the inactive state is consistent with coronal emission from the secondary late type star.
\end{abstract}

Key words. accretion, accretion disks - stars: binaries: close - stars: novae, cataclysmic variables - stars: individual: AM Her - X-rays: stars

\section{Introduction}

AM Her is the prototype of Polars, strongly magnetic Cataclysmic Variables (mCVs) (10-230 MG), and consists of a magnetized $(\sim 14 \mathrm{MG})$ white dwarf accreting from a late type (M4V) Roche lobe-filling secondary star. Polars are characterized by long-term (months to years) high and low accretion states which, due to the absence of an accretion disc in these systems, reflect changes in the mass loss rate of the donor star. AM Her is the brightest and best monitored Polar in the optical range and hence represents a test object to study the evolution of the instantanous mass accretion rate with time and then to understand the causes of the mass transfer variations from the secondary star. Bright and faint luminosity states $(\Delta V \sim 2-3 \mathrm{mag}$ ) occur on irregular timescales from less than a day to months. Different models have been discussed to account for the long-term mass transfer variations in CVs (Livio \& Pringle 1994; King \& Cannizzo 1998) and the occurrence of starspots at the inner Lagrangian point appears to be the most likely explanation. Along this line, Hessman et al. (2000) derived the mass transfer rate history of AM Her, using the long term optical variability and convert it into starspot filling

Send offprint requests to: D. de Martino,

e-mail: demartino@na.astro.it factors. They conclude that the density of the starspots near the L1 point is unusually high (about $50 \%$ ).

On the other hand, monitoring of the X-ray activity has been relatively sparce, but has already brought further insights into the variability of the mass accretion rate. In particular, since its launch, we undertook a programme with the BeppoSAX satellite to monitor the X-ray behaviour of AM Her, to infer the evolution of the X-ray luminosity and its X-ray spectral variability. In this work we present new and most recent observations of AM Her carried out during the life of BeppoSAX together with simultaneous optical photometry acquired at the Loiano Bologna Observatory, which reveal further new results on the accretion variability and on the possible identification of the coronal X-ray emission from the magnetically active secondary star.

\section{Observations and data reduction}

During its life the BeppoSAX satellite (Boella et al. 1997) performed with the co-aligned Narrow Field Instruments (NFI) four pointed observations of AM Her. The source was found to be in different states as summarized in Table 1 and depicted in Fig. 1, where the secular AAVSO optical light curve is shown along with the times of the X-ray pointings. 
Table 1. History of BeppoSAX observations of AM Her and log of optical observations in 2001.

\begin{tabular}{|c|c|c|c|}
\hline Date & $\begin{array}{c}\text { MECS Exp. Time } \\
\text { (s) }\end{array}$ & Flux $^{1} /$ Count Rate ${ }^{2}$ & Notes \\
\hline Sep. 6, 1997 & $24700^{3}$ & $0.18 / 0.049$ & Active state $^{a}$ \\
\hline Sep. 6, 1997 & & $0.024 / 6.5 \times 10^{-3}$ & Quiescent low state ${ }^{a}$ \\
\hline May 8, 1998 & 33500 & $1.80 / 0.22$ & Intermediate state ${ }^{b}$ \\
\hline Aug. 12, 1998 & 80600 & $12 / 1.35$ & High state ${ }^{b}$ \\
\hline Apr. 22, 2001 & $30500^{3}$ & $1.05 / 0.135$ & Active state ${ }^{c}$ \\
\hline Apr. 22, 2001 & & $0.0154 / 4.5 \times 10^{-3}$ & Quiescent low state ${ }^{c}$ \\
\hline Date & Filter & $\begin{array}{c}\text { Exp. Time } \\
\text { (s) }\end{array}$ & $\begin{array}{l}\text { Tintegration } \\
\text { (s) }\end{array}$ \\
\hline \multirow[t]{4}{*}{ Apr. 22, 2001} & $\bar{U}$ & 11300 & 15 \\
\hline & $B$ & 11300 & 5 \\
\hline & $V$ & 11250 & 10 \\
\hline & $R$ & 11250 & 20 \\
\hline
\end{tabular}

${ }^{1} 2-10 \mathrm{keV}$ phase averaged flux in units of $10^{-11} \mathrm{erg} \mathrm{cm}^{-2} \mathrm{~s}^{-1}$, see references for best fit models.

${ }^{2}$ Count rates in units of $\mathrm{cts} \mathrm{s}^{-1}$.

${ }^{3}$ Total on source exposure time.

${ }^{a}$ de Martino et al. (1998).

${ }^{b}$ Matt et al. (2000).

${ }^{c}$ This work.

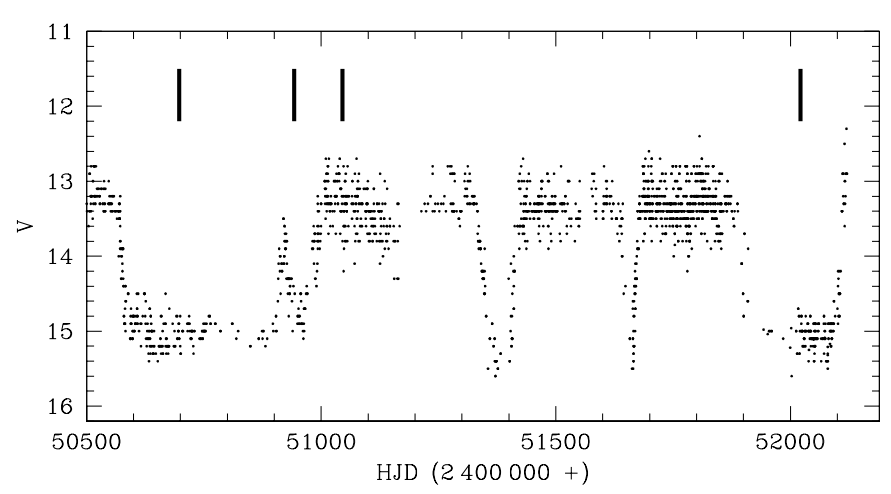

Fig. 1. The long term optical light curve from AAVSO data. The times of BeppoSAX NFI pointings are marked with vertical lines.

\subsection{The BeppoSAX data}

The last BeppoSAX pointing of AMHer was carried out on April 22, 2001 during a low state that started in December 2000 and terminated at the end of July 2001 (Fig. 1). It was detected at its lowest level by the Low Energy Concentrator Spectrometer (LECS) [0.1-10 keV] and by the Medium Energy Concentrator Spectrometer (MECS) [1.3-10 keV] with an effective on source exposure time of $15.5 \mathrm{ks}$ and $30.5 \mathrm{ks}$ respectively.

Spectra and light curves from the MECS and LECS instruments have been extracted from a circular region with a radius of $3^{\prime}$ and $2^{\prime}$ respectively, using the Ftools XSELECT procedure. Background data were extracted from blank sky pointings using the same radius and subtracted from the data. The BeppoSAX observation initially shows AMHer at a higher count rate level (henceforth "active state”) which was monitored for $\sim 2.2 \mathrm{hr}$, followed by a deep low count rate state monitored for $\sim 16.1 \mathrm{hr}$ (henceforth “quiescent state”). In Fig 2, the LECS and MECS light curves show a decrease by a factor of 47 and 30 in count rates between the active and quiescent states respectively.

\subsection{The optical data}

Optical $U B V R$ photometry was carried out at the $1.5 \mathrm{~m}$ telescope of Loiano-Bologna Observatory (Italy) with a three channel photometer (TTCP) on the same day of BeppoSAX observation, covering simultaneously the quiescent state for $\sim 3.14 \mathrm{hr}$ (Fig. 2, upper panel). Data reduction was performed using the TTCP software developed at the Astronomical Observatory of Capodimonte-Naples. A set of Landolt standard stars were observed to calibrate data resulting in an average $U=14.43 \pm 0.04 \mathrm{mag}, B=15.43 \pm 0.1$ and $V=$ $14.99 \mathrm{mag} \pm 0.04 \mathrm{mag}$. $R$ band data were not calibrated due to unadequate detector efficiency estimates and poor calibration data. The optical level during these observation is similar to that observed during the low state in September 1997 $(V=15.08 \mathrm{mag})$.

\section{Data analysis and results}

The observations were analyzed separately according to the BeppoSAX count rate level.

\subsection{The $X$-ray active state}

During the active state AMHer shows a first peak or flare followed by a second one and finally a fast exponential 


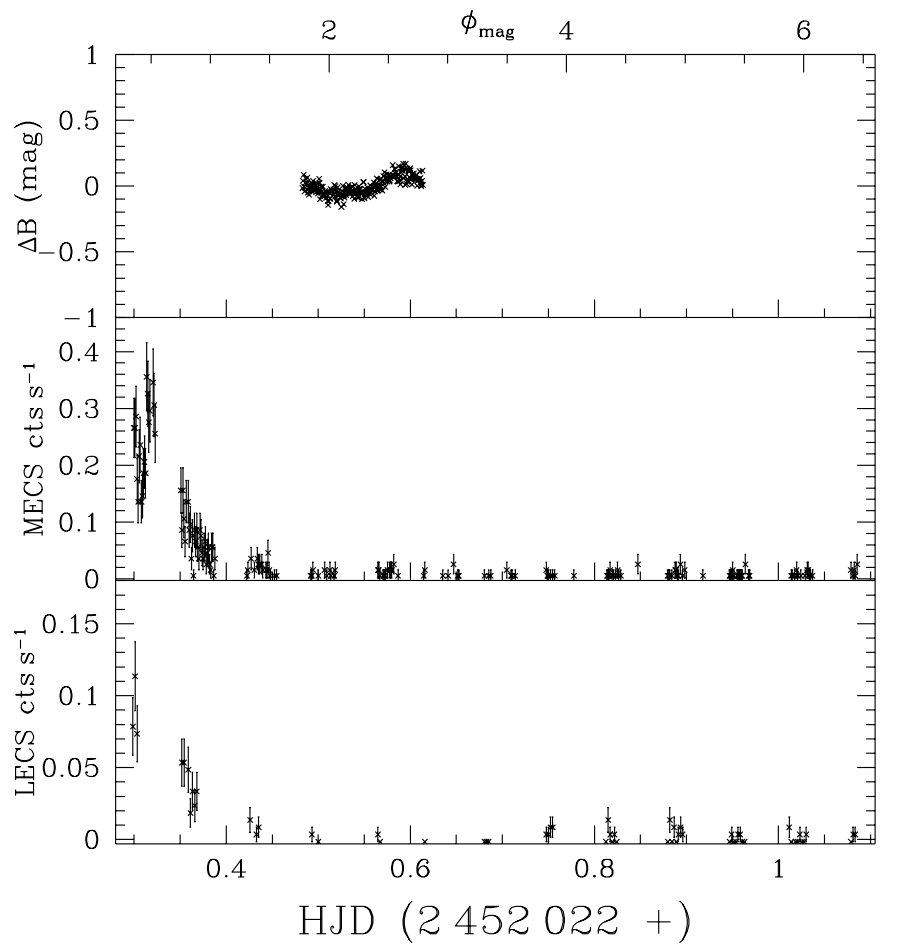

Fig. 2. The light curves from the LECS (lower panel), the MECS (middle panel) instruments. Gaps are due to the BeppoSAX satellite orbit. The $B$ band differential photometry acquired simultaneously is shown in the upper panel. Magnetic phases are also reported on the top.

decay ( $\propto \mathrm{e}^{-t / \tau}$, with $\tau=51 \mathrm{~min}$, in the MECS detector). Due to the lower efficiency, the LECS coverage is poorer. A similar fast decay was recorded by BeppoSAX during the low state in September 1997 (de Martino et al. 1998), but at that time the active state count rate level was three times lower than the one observed in April 2001.

We have folded the MECS active state 2001 data along the $3.1 \mathrm{hr}$ rotational/orbital period using the magnetic ephemeris reported in Heise \& Verbunt (1988), and compared with the high state light curve observed by BeppoSAX in 1998 (Matt et al. 2000). Although fragmentary, the light curve shown in Fig. 3 shows that the two peaks find their counterparts in the high state light curve. They are consistent with the bright phase (maximum) and hence with the X-ray emission from the main accreting pole which is self-occulted at $\phi_{\text {mag }}=0.1-0.3$. However the decay occurs somewhat earlier (by $\sim 0.1$ in phase), possibly suggesting the concurrence of the beginning of disappearance of the main accreting pole and of an intrinsic drop in the accretion flux. It is worth noting that the X-ray active state observed during September 1997 was also compatible with Xray emission onto the main accreting pole (de Martino et al. 1998).

The averaged combined LECS and MECS spectrum during the active state does not require a black-body component, similar to that of September 1997. The spectrum was then fitted using the XSPEC package with a MEKAL model with iron abundances fixed at the solar value (Matt et al. 2000) plus absorption fixed at $N_{\mathrm{H}}=9 \times 10^{19} \mathrm{~cm}^{-2}$ (Gänsicke et al. 1995),

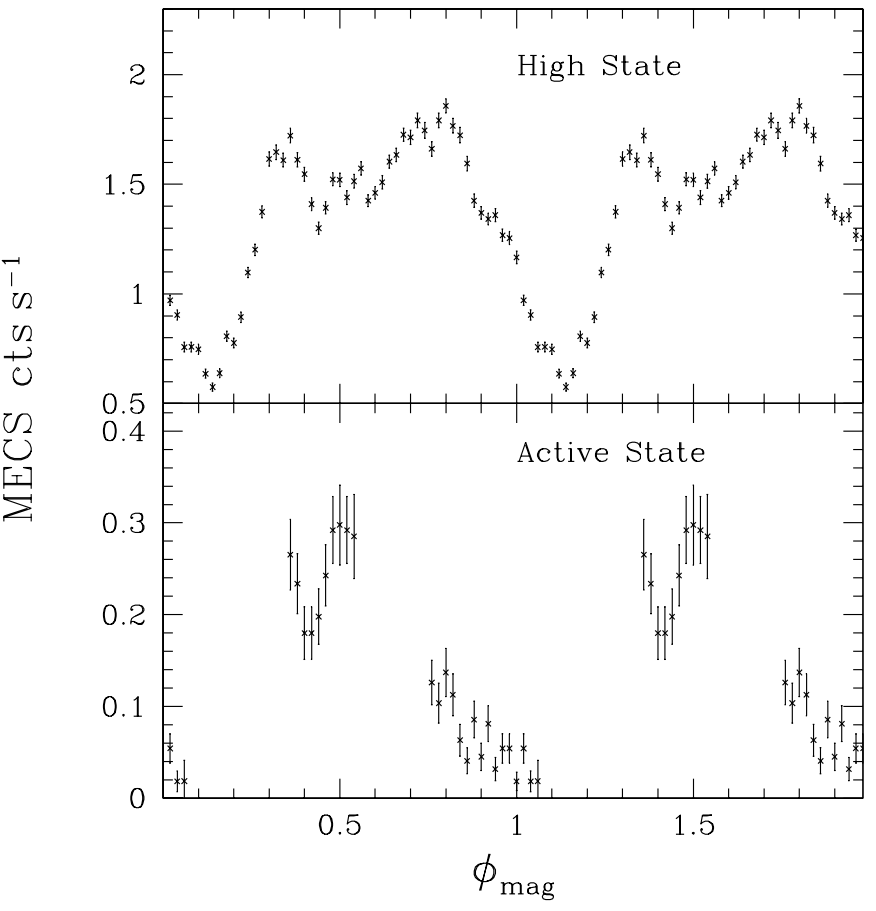

Fig. 3. The MECS folded light curve of the active state (lower panel) and that of the high state in 1998 (Matt et al. 2000) (upper panel) shown for comparison (see text).

which gives $k T=14_{-6}^{+23} \mathrm{keV}\left(\chi_{\text {red }}^{2}=1.38\right.$ for 44 d.o.f.). Though the fit is poor (Fig. 4, left panel), the optically thin plasma temperature is compatible with that found during intermediate and high states (Matt et al. 2000). The data are not of enough quality to allow a partial covering component to be added. The bolometric flux during the active state is $2.6 \times 10^{-11} \mathrm{erg} \mathrm{cm}^{-2} \mathrm{~s}^{-1}$.

\subsection{The quiescent state}

\subsubsection{The X-ray emission}

While during the active state, the count rate is larger than that observed in the September 1997 active state, this is not the case for the quiescent state count rate, which, in September 1997, was $\sim 1.4$ times higher than the one recorded in 2001, thus making the present BeppoSAX data the faintest X-ray state detected so far in AM Her.

No orbital modulation can be detected in the MECS data, thus suggesting that accretion onto the main pole has switched off.

The quiescent state average combined LECS and MECS spectrum (Fig. 4, right panel) was fitted with the same MEKAL plus absorption model which gives a much lower temperature $k T=1.5 \pm_{-0.7}^{+0.9} \mathrm{keV}\left(\chi_{\text {red }}^{2}=1.43\right.$, for 24 d.o.f. $)$, the lowest determined so far in AMHer. The bolometric flux is $1.5 \times 10^{-12} \mathrm{erg} \mathrm{cm}^{-2} \mathrm{~s}^{-1}$.

\subsubsection{The optical quiescent light curves}

Unlike the X-rays, the optical light curve shows an orbital modulation (Fig. 2). Folding differential photometric $U B V R$ data 

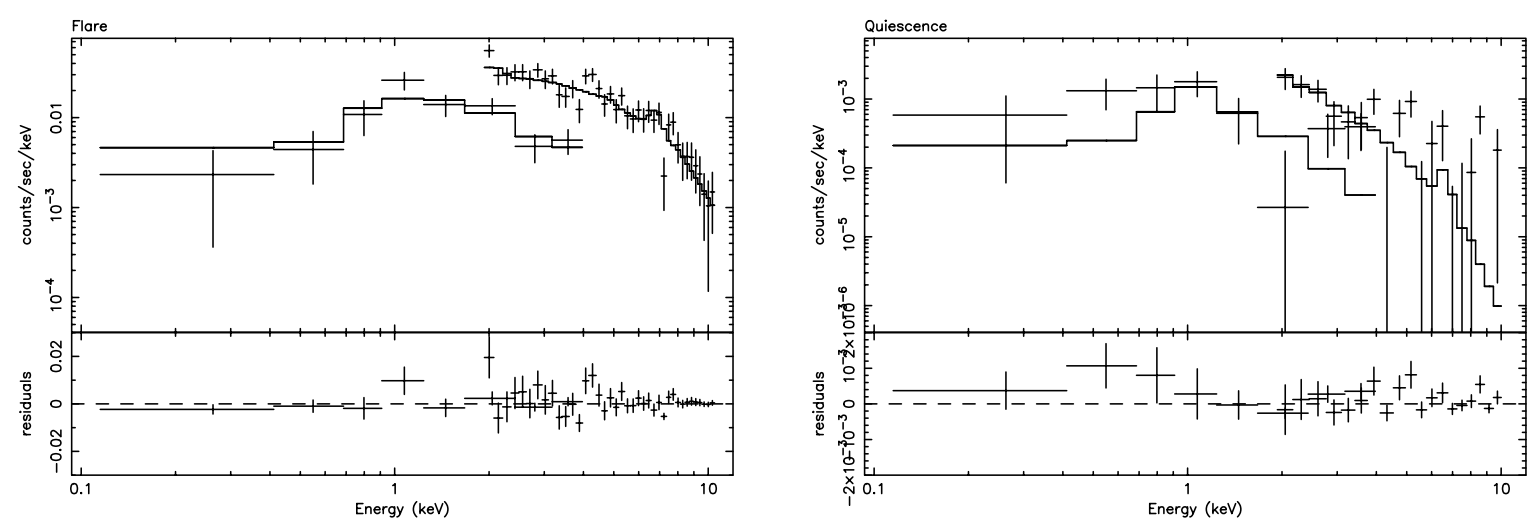

Fig. 4. The combined LECS and MECS spectra during the active state (left panel) and the quiescent state (right panel) fitted with MEKAL models (see text).

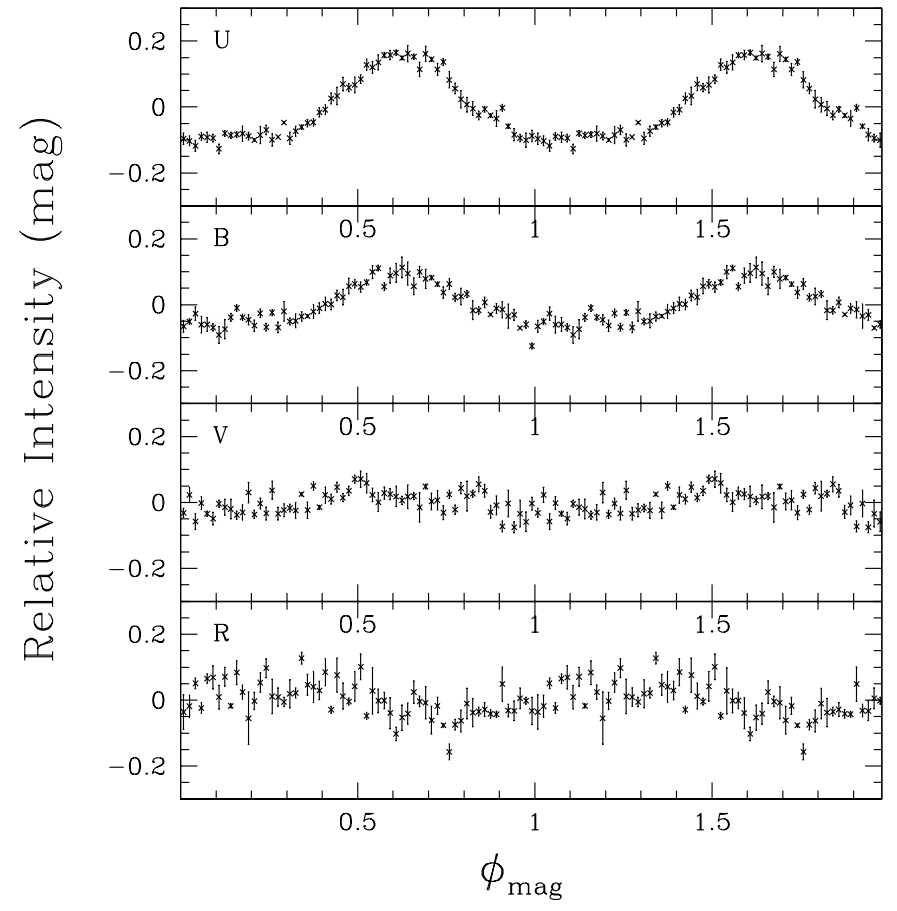

Fig. 5. The differential $U B V R$ folded light curves obtained simultaneously during the X-ray quiescent state.

along the orbital period, a clear colour dependence is found, as depicted in Fig. 5. The amplitude of modulation changes from $20 \%$ in the $U$ band, to $13 \%$ in the $B$ and is almost absent in $V$ and $R$ bands. The blue filters show the typical behaviour observed in the far-UV range (Gänsicke et al. 1995; Gänsicke et al. 1998) with a maximum at $\phi_{\mathrm{mag}}=0.64$ and a minimum at $\phi_{\mathrm{mag}}=0.1$. This is consistent with the emission from the white dwarf plus the heated pole cap which dominates the UV range during both high and low accretion states (Gänsicke et al. 1995). The lack of significant modulation in $V$ and $R$ bands suggests that cyclotron emission, known to be dominant at these wavelengths, is negligible during this low state.

Following the method described in Gänsicke et al. (2001), we have attempted to model the calibrated $U B V$ light curves. The first attempt assumes that the $U$ band light curve is due entirely to emission from the white dwarf plus the heated polar spot. We fix the following parameters to the values derived from the previous works (Gänsicke et al. 1995, 1998): distance $d=90 \mathrm{pc}$, the white dwarf radius $R_{\mathrm{wd}}=1 \times 10^{9} \mathrm{~cm}$, inclination $i=50^{\circ}$, colatitude and azimuth of the polar cap $\beta=55^{\circ}$ and $\Psi=0^{\circ}$. Cyclotron emission is neglected as well as the emission from the accretion stream. The secondary M4V star contribution is taken into account as described in Gänsicke et al. (2001) and set to $U=19.76 \mathrm{mag}, B=19.43 \mathrm{mag}$ and $V=16.83 \mathrm{mag}$. The temperature of the white dwarf is then adjusted to match the faint-phase flux which agrees with the UV IUE low state results (Gänsicke et al. 1995). The central spot temperature and the spot opening angle are then adjusted to match the amplitude and width of the observed modulation in the $U$ band, thus giving $T_{\mathrm{wd}}=19000 \mathrm{~K}, T_{\text {spot }}=50000 \mathrm{~K}, \Theta_{\text {spot }}=20^{\circ}$. The model, however, overpredicts the observed $B$ band flux and underpredicts the $V$ flux.

A match with the observed light curves is found, as shown in Fig. 6, by lowering the temperature $\left(T_{\mathrm{wd}}=17000 \mathrm{~K}\right)$ of the white dwarf in order to decrease its contribution. The spot temperature and opening angle are the same as before since they represent the amplitude and shape of the modulation well. However, a constant component (with $U=16.1 \mathrm{mag}$ ) has to be added. This could be attributed to the optically thin emission of a weak and faint accretion stream. Also, a very weak $V=18.5 \mathrm{mag}$ component is required to match the $V$ flux. This could be due to a faint contribution from cyclotron emission still present. The lack of a clear modulation in the $R$ band light curve (Fig. 5) is not easy to interpret, although the data cannot exclude that a modulation at a $10 \%$ is present. In the red, ellipsoidal variations from the secondary star of about $0.1-0.2 \mathrm{mag}$ are expected (Bailey et al. 1988) together with the heated white dwarf atmospheric spot modulation. A possibility could be due that the weak cyclotron emission, whose behaviour is in antiphase with the white dwarf spot modulation (see Gänsicke et al. 2001 for modeling of cyclotron contribution), is present producing a flat $R$ band light curve. Unfortunately the lack of reliable calibration in this band does not allow us to properly assess this issue.

\section{Discussion and conclusions}

The last pointed BeppoSAX observation of AM Her caught the source during its deepest low state ever observed. The X-ray 


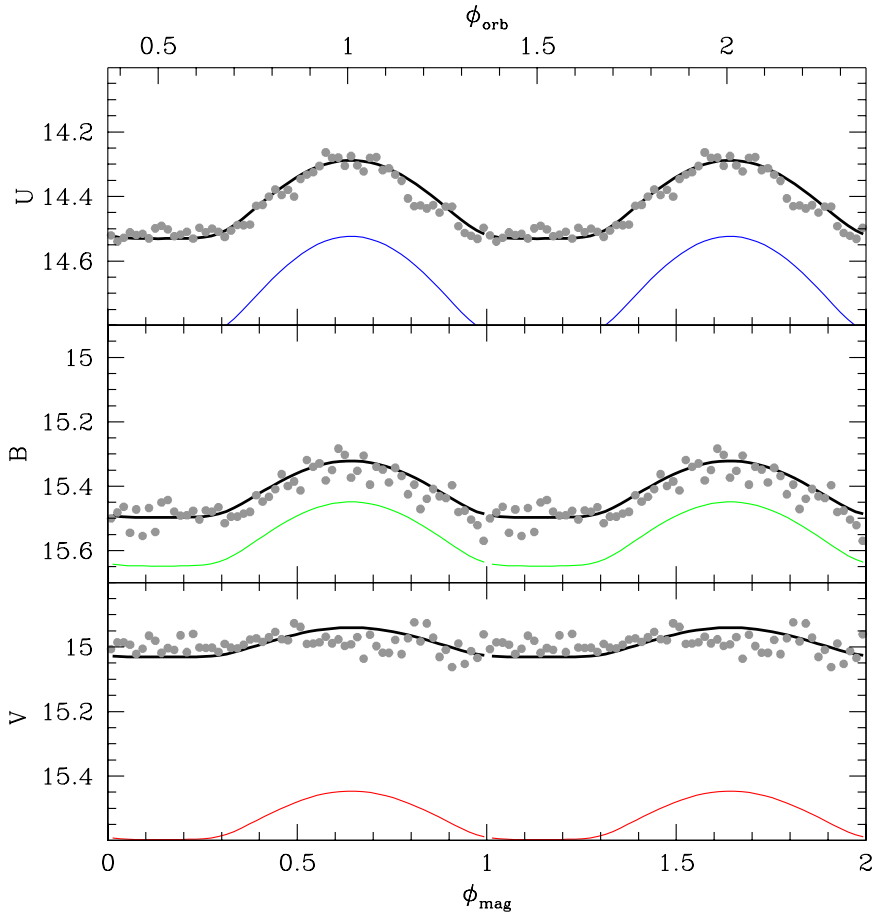

Fig. 6. The observed $U B V$ light curves during the X-ray quiescent state together with the fitted model as described in the text. The solid thick line is the sum of all contributions from the heated white dwarf spot at $50000 \mathrm{~K}$, the un-heated white dwarf at $17000 \mathrm{~K}$ plus a constant contribution from the accretion stream. In the $V$ band an additional weak $18.5 \mathrm{mag}$ component is included. The solid thin line represents the heated white dwarf spot. The other contributions are not shown due to the large magnitude differences. See text for details.

flux shows a rapid variability from an active to a quiescent state similar to that observed during another prolonged low state in 1997 (de Martino et al. 1998). Unfortunately, also for this second time, AM Her was bright at the beginning of the observation and, although the bright state is consistent with X-ray accretion-induced emission, it is not possible to assess whether this was a temporary accretion event or if the source was previously in a constant accretion epoch. However, the drop in $\mathrm{X}$-ray flux indicates that the accretion rate decreased by a factor of $\sim 17$ in less than one hour. A very rapid variation (18 min) in the X-ray flux also has been recorded in XMM-Newton data of UZ For during a deep low X-ray state and interpreted as an accretion event (Still \& Mukai 2001). For the active state, we derive an estimate of the accretion luminosity, assuming that about half of the thermal bremsstrahlung and cyclotron radiation emitted from the post-shock region is intercepted by the white dwarf and re-emitted in the UV $\left(L_{\mathrm{UV}}=L_{\mathrm{tb}}+L_{\mathrm{cyc}}\right)$ and neglecting the contribution of a re-processed component in the EUV range (cf. Gänsicke et al. 1995 and similar reasoning in de Martino et al. 1998). We then estimate an accretion rate of $4.9 \times 10^{-11} M_{\odot} \mathrm{yr}^{-1}$. The drop in mass accretion rate therefore indicates that the $\mathrm{X}$-ray emission during the quiescent state is not due to accretion. The much lower temperature $(1.5 \mathrm{keV})$ is compatible with coronal temperatures of M type dwarfs (Schmitt et al. 1990) as well as the low X-ray luminosity in the $0.05-3 \mathrm{keV}$ of $8.6 \times 10^{29} \mathrm{erg} \mathrm{s}^{-1}$ is compatible with those observed in active late type stars
(Pallavicini et al. 1990). Furthermore the emission measure derived during the quiescent state $E M=4.6 \times 10^{52} \mathrm{~cm}^{-3}$ is also consistent with coronal values. We have also compared the $\mathrm{X}$-ray luminosity during the quiescent state with that expected from coronal emission of rapidly rotating late type stars. For a secondary star filling its Roche lobe in a $3.09 \mathrm{hr}$ orbital period binary, the period-radius relation (Patterson 1984) gives for the secondary star $R_{\mathrm{sec}}=0.32-0.36 R_{\odot}$. The predicted saturation value for the X-ray luminosity due to rotation is $\propto R_{\mathrm{sec}}^{2}$ (Fleming et al. 1989), corresponding to $4.9-6.3 \times 10^{29} \mathrm{erg} \mathrm{s}^{-1}$ (0.3-3 keV band), in remarkable agreement with the luminosity derived in the same range for the BeppoSAX quiescent state $\left(6.4 \times 10^{29} \mathrm{erg} \mathrm{s}^{-1}\right)$.

Moreover, the lack of $V$ and $R$ band modulations indicate that, even if present, cyclotron emission is very weak, thus implying that AM Her really switched-off accretion during the BeppoSAX observation. All this indicates that the $\mathrm{X}$-ray emission can be indeed and more safely ascribed to the secondary star than done previously for the quiescent state in September 1997.

As for the 1997 BeppoSAX data set, still remains unclear the cause of the rapid drop of accretion flux, with timescales remarkably close to the dynamical timescale of the secondary star. The observations of a rapidly evolving burst in UZ For observed with XMM-Newton further confirms that such rapid changes occur in AMHer stars and a coronal mass ejection event at the L1 point may be the cause of the observed active state (cf. de Martino et al. 1998 for discussion). It is clear that secondaries in these close binary systems are still far from being understood and further X-ray observations during low states would help in assessing the nature of these active secondary stars.

Acknowledgements. In this work, we have used and gratefully acknowledge the data from the AAVSO International Database, based on observations submitted to the AAVSO by variable star observers worldwide. DdM and GM acknowledge financial support from ASI. BTG was supported by a PPARC advanced Fellowship.

\section{References}

Bailey, J., Hough, J. H., \& Wickramasinghe, D. T. 1988, MNRAS, 23, 1988

Boella, G., Butler, R. C., Perola, G. C., et al. 1997, A\&AS, 122, 299

de Martino, D., Gänsicke, B. T., Matt, G., et al. 1998, A\&A, 333, L31

Fleming, T. A., Gioia, I. M., \& Maccacaro, T. 1989, ApJ, 340, 1011

Gänsicke, B. T., Buermann, K., \& de Martino, D. 1995, A\&A, 303, 127

Gänsicke, B. T., Hoard, D. W., Beuermann, K., et al. 1998, A\&A, 338, 933

Gänsicke, B. T., Fisher, A., Silvotti, R., \& de Martino, D. 2001, A\&A, 372,557

Heise, J., \& Verbunt, F. 1988, A\&A, 189, 112

Hessman, F. V., Gänsicke, B. T., \& Mattei, J. 2000, A\&A, 361, 952

King, A. R., \& Cannizzo, J. K. 1998, ApJ, 499, 348

Livio, M., \& Pringle, J. E. 1994, ApJ, 427, 956

Matt, G., de Martino, D., Gänsicke, B. T., et al. 2000, A\&A, 358, 177

Pallavicini, R., Tagliaferri, G., \& Stella, L. 1990, A\&A, 228, 403

Patterson, J. 1984, ApJS, 54, 443

Schmitt, J. H. M. M., Collura, A., Harnden, F. R., et al. 1990, ApJ, 365, 704

Still, M., \& Mukai, K. 2001, ApJ, 562, L71 